\title{
Wavelet Based Some Julia Sets of Rational Maps Having Zhukovskii Function
}

\author{
Jean Bosco Mugiraneza \\ Department of Computer Science, Kigali Independent University \\ P. O. Box 2280, Kigali-Rwanda, mjbosco8@hotmail.com
}

\begin{abstract}
The dynamics of rational maps and their properties are interesting because of the presence of poles and zeros. In this paper we have computed Julia sets of rational maps having Zhukovskii Function for which the double of the first derivative has no Herman rings. The data points out of the Julia set in Matlab workspace were imported to Matlab Signal Processing Tool for their analysis. We have sampled the data points with the sampling frequency of $8192 \mathrm{~Hz}$ and obtained complex signals. We have then applied the band pass filter to these complex signals. The effect of the band pass filter has generated complex analogue modulated signals.
\end{abstract}

Index Terms - Wavelets, Julia Set, Maps, Matlab SPTool, Zhukovskii Function

\section{INTRODUCTION}

The study of iterated holomorphic mappings began in the $19^{\text {th }}$ century but only came to flower in the $20^{\text {th }}$. In this section our objective is to discuss basic definitions and tools used in complex dynamics. Section 2 provides the mapping properties of the rational map having Zhukovskii Function, section 3 discusses wavelets based Iterated Functions, section 4 presents simulation results of computer generated images using Matlab SPTool, we will wind with the conclusion. The reader needs to possess introductory background in complex analysis.

A rational map is defined by a ratio of two polynomials,

$R(z)=\frac{P(z)}{Q(z)}=\frac{p_{n} z^{n}+p_{n-1} z^{n-1}+\ldots+p_{0} z^{0}}{q_{m} z^{m}+q_{m-1} z^{m-1}+\ldots+q_{0} z^{0}}$

where $n, m \geq 0, p_{n} \neq 0$ and $q_{m} \neq 0 ; p_{i}, q_{i} \in C$.

The algebraic degree of

$R=\max (\operatorname{deg} P, \operatorname{deg} Q)=\max (n, m)$.

If $Q(z)=(z-a)^{k} s(z), k \notin N$ and $s(a) \neq 0$, then $R$ is said to have a pole of order $k$ at $a$. The complex number $z_{0}$ is said to be a critical point of $R$ if either $R^{\prime}\left(z_{0}\right)=0$ or if $\left(z-z_{0}\right)^{k}, k \geq 2$, is a factor of $Q(z)$. The image of $z_{0}$ under $R, R\left(z_{0}\right)$, is called a critical value of $R$, in the case that $\left(z-z_{0}\right)^{k}$ is a factor of $Q, R\left(z_{0}\right)=\infty$. Infinity is a critical point of $R$, if $R^{\prime}(\infty)=0$. The presence of a pole at the origin reveals that there is a neighborhood of the origin that is mapped to the basin at infinity [1].

A rational map of $R$ degree $d \geq 2$ has $2 d-2$ critical points counted with multiplicity. This comes from Riemann-Hurwitz formula. It has $d+1$ fixed points counted with multiplicity. This is an instance of Lefschetz fixed point formula [2]. We are interested in finding the fixed points because fixed points of a given function are interesting in analyzing the function about its equilibrium point. A fixed point also known as an invariant point of a function is a point that is mapped to itself by the function. That is to say, if $z_{0} \in C_{\infty}$ is a fixed point of a function then $R\left(z_{0}\right)=z_{0}$. The derivative of $R$ evaluated at $z_{0}, R^{\prime}\left(z_{0}\right)=\lambda$, is sometimes referred to as the multiplier or the eigenvalue for the fixed point of $R$ at $z_{0}$. The fixed point is classified based on the value of $|\lambda|$.

If $|\lambda|>1$, then $z_{0}$ is repelling fixed point.

If $0<|\lambda|<1$, then $z_{0}$ is an attracting fixed point.

If $|\lambda|=0$, then $z_{0}$ is a superattracting fixed point.

If $|\lambda|=1$, then $z_{0}$ is an indefferent fixed point.

If $z_{0}$ is neutral or indifferent, we write $f^{\prime}\left(z_{0}\right)=e^{2 \pi i \theta_{0}}$; further if $\theta_{0}$ is rational, then $z_{0}$ is rationally indifferent or parabolic, otherwise $z_{0}$ is irrationally indifferent.

Assume that $z_{0}$ is an attracting fixed point of $R$ and $|\lambda|<\rho<1$,

then $\left|R(z)-R\left(z_{0}\right)\right|=\left|R(z)-z_{0}\right| \leq \rho\left|z-z_{0}\right|$

neighborhood $D$ of $z_{0}$.

Then

$\left|R^{n}(z)-R^{n}\left(z_{0}\right)\right|=\left|R^{n}(z)-z_{0}\right| \leq \rho^{n}\left|z-z_{0}\right|$ and $R^{n}(z) \rightarrow z_{0}$ uniformly on $D$. The basin of attraction of $z_{0}, A\left(z_{0}\right)=\left\{z \in C_{\infty}: R^{n}(z) \rightarrow z_{0}\right\}$. 
It is well known that the dynamics of a rational map is very much influenced by the forward orbits of its critical points [3]. The forward orbit $E$ of a periodic point is called a cycle, because $R / E$ is a cyclic permutation.

The Julia set can be defined as the closure of the set of repelling periodic points for $R$. Here a point $z$ is periodic if $R^{p}(z)=z$ some $p>0$; it is

repelling if $\left|\left(R^{p}\right)^{\prime}(z)\right|>1$

indifferent if $\left|\left(R^{p}\right)^{\prime}(z)\right|=1$; and

attracting if $\left|\left(R^{p}\right)^{\prime}(z)\right|<1$.

We can characterize a cycle of order $n$ similarly, based on the value of the derivative of $R^{n}$ at some points in the cycle. First we note that

$$
\begin{aligned}
& \left(R^{p}\right)^{\prime}\left(z_{0}\right)=R^{\prime}\left(R^{n-1}\left(z_{0}\right)\right) R^{\prime}\left(R^{n-2}\left(z_{0}\right)\right) . . R^{\prime}\left(z_{0}\right) \\
& =R^{\prime}\left(\left(z_{n-1}\right)\right) R^{\prime}\left(R^{\prime}\left(z_{n-2}\right)\right) \ldots R^{\prime}\left(z_{0}\right)
\end{aligned}
$$

any point in the cycle, and we can characterize a cycle (and the periodic points in the cycle) as repelling, attracting, superattracting, rational indefferent, or irrational indeferent according to the nature of the fixed point $z_{0}$ for the map $R^{n}$. All attracting and superattracting fixed points and cycles are a part of the Fatou set. This follows because in a small neighborhood around an attracting (superattacting) fixed point, the map $R(z)$ is a contraction [4]. Rational maps are complex analytic, so a broad spectrum of techniques can contribute to their study (quasiconformal mappings, potential theory, algebraic geometry, etc.).The rational maps of a given degree form a finite dimensional manifold, so exploration of this parameter space is especially tractable [5].

Let $R(z)=\frac{P(z)}{Q(z)}$ where $P$ and $Q$ are polynomials with $\operatorname{deg} P>\operatorname{deg} Q$. In this case $|R(z)| \rightarrow \infty$ as $z \rightarrow \infty$ so infinity is regarded as a fixed point. Also, infinity is an attracting fixed point, in the following two cases:

1. $\operatorname{deg} P>\operatorname{deg} Q+1$

Then the Julia set of $R$ is the boundary of the basin of attraction of infinity.

2. $\operatorname{deg} P>\operatorname{deg} Q+1$ and $\alpha=\left|\frac{p_{n}}{q_{m}}\right|<1 \quad$ where $q_{m}$ is the leading coefficient of $Q$ and $p_{n}$ is the leading coefficient of $P$. If $\alpha=\left|\frac{p_{n}}{q_{m}}\right|>1$ then infinity is not an attracting fixed point of $R$.
If $\alpha=\left|\frac{p_{n}}{q_{m}}\right|=1$ then infinity is a neutral fixed point of $R$.

For Julia sets of rational maps with numerator not of higher degree than denominator, then infinity is not a fixed point.

\section{Theorem 1.1}

If a rational map has only one fixed point which is repelling or has multiplier 1 , then the Julia set is connected.

\section{MAPPING PROPERTIES}

In [6] the family of the maps $G_{\lambda}(z)=z^{n}+\frac{\lambda}{z^{d}}$ with $n \geq 2, d \geq 1$ was investigated and the most complicated case was when $n=d=2$. These maps reduce to special case $z \mapsto z^{n}$. So $G_{0}(z)=z^{n}$ is a polynomial of degree $n$, there is a superattracting fixed point at the origin (when $n \geq 2$. ), and the Julia set converges to the unit disk as $\lambda \rightarrow 0$. In our paper we attempt to study the class of rational maps, $R_{\alpha, n}(z)=\frac{1}{2}\left(z+\frac{\alpha}{z^{n}}\right)$ for $n \geq 0$.

\subsection{Polynomials of First Degree}

The rational map $R_{\alpha, n}(z)=\frac{1}{2}\left(z+\frac{\alpha}{z}\right)$ reduces to a polynomial of degree one.

- If $\alpha=0$, then $R_{0, n}(z)=\frac{1}{2} z$ and fixes two points in extended complex plane $C \cup \infty$ and is similar to a scaling.

- If $n=0, R_{\alpha, 0}(z)=\frac{1}{2} z+\frac{1}{2} \alpha$ and fixes two points in extended complex plane $C \cup \infty$ and is similar to a scaling followed by translation.

The above conditions are special cases of Möbius transformation.

\subsection{Zhukovskii Function}

Our goal in this section is to discuss another special case of the map $R_{\alpha, n}(z)=\frac{1}{2}\left(z+\frac{\alpha}{z^{n}}\right)$ given by $T(z)=\frac{1}{2}\left(z+\frac{1}{z}\right)$ 
this is Zhukovskii map, and it finds applications in fluid dynamics [7]. Suppose that $z=\rho e^{i \theta}$, then

$$
\begin{aligned}
& \operatorname{Re}(T(z))=x=\frac{1}{2}\left(\rho+\frac{1}{\rho}\right) \cos \theta \\
& \operatorname{Im}(T(z))=y=\frac{1}{2}\left(\rho-\frac{1}{\rho}\right) \sin \theta
\end{aligned}
$$

Eliminating $\theta$ by squaring both sides of these equations and adding them leads to

$$
\frac{4 x^{2}}{\left(\rho+\frac{1}{\rho}\right)^{2}}+\frac{4 y^{2}}{\left(\rho-\frac{1}{\rho}\right)^{2}}=1
$$

Eliminating $\rho$ in the same manner gives

$$
\frac{x^{2}}{\cos ^{2} \theta}-\frac{y^{2}}{\sin ^{2} \theta}=1
$$

\section{Lemma 2.1}

$$
\text { Zhukovskii map } T(z)=\frac{1}{2}\left(z+\frac{1}{z}\right) \text { maps circles }
$$

centered at the origin onto ellipses (Zhukovskii ellipses) and lines through the origin onto hyperbolas

(Zhukovskii hyperbolas). The map $z \rightarrow z^{2}$ takes a Zhukovskii ellispse to an ellipse with one focus at the origin. It takes a Zhukovskii hyperbola to one branch of a hyperbola that has the origin as its focus, and a line to a parabola with focus at the origin [8].

It follows that

$$
\begin{aligned}
(T(z))^{2} & =\frac{1}{2}\left[T\left(z^{2}\right)+1\right] \\
(T(i z))^{2} & =-\frac{1}{2}\left[T\left(z^{2}\right)-1\right]
\end{aligned}
$$

Adding Eqn. (2.6) and Eqn. (2.7) we get

$$
(T(z))^{2}+(T(i z))^{2}=1
$$

$$
\text { In particular, }(T(1))^{2}+(T(i))^{2}=1 \text {, }
$$

since $T(1)=1$ and $T(i)=0$.

We can now change the form of Zhukovskii function in Eqn. (2.1) to consider the family

$$
T_{\delta}(z)=\frac{\delta}{2}\left(z+\frac{1}{z}\right)
$$

The Julia set of $T_{\delta}(z)$ is defined as to be the set of points for which the family of iterates of $T_{\delta}(z)$ is not a normal family in the sense of Montel. We denote the Julia set by $J\left(T_{\delta}\right)$. For each $\delta$ the map $T_{\delta}(z)$ has two critical points given by -1 and +1 . The critical values are given by $-\delta$ and $+\delta$. Since $T_{\delta}(-z)=T_{\delta}(-z)$, it follows that the orbits of these critical points are symmetric with respect to $z \mapsto-z$. The orbits of the critical points are called the critical orbits. The behavior of the critical orbits of a complex map determines to a large extent the dynamics of the map on the whole Riemann sphere [9]. $T_{\delta}(z)$ has fixed points at $z= \pm \sqrt{\frac{-\delta}{\delta-2}}$ and $T_{\delta}^{\prime}(z)=2 \delta-2$, so $T_{\delta}(z)$ has an attracting fixed point when $\delta$ lies in the open disk of radius 1 centered at 1 .

$T_{\delta}(i z)=i \frac{\delta}{2}\left(z-\frac{1}{z}\right)$

This means that the imaginary axis is invariant under $T_{\delta}$.

\subsection{Basic Mapping Properties}

If $n$ is odd, then $R_{\alpha, n}(-z)=-R_{\alpha, n}(z)$, this means that the map presents some symmetry with respect to the origin. Since the rational map $R_{\alpha, n}(z)=\frac{1}{2}\left(z+\frac{\alpha}{z^{n}}\right)$ has the numerator of higher degree than that of the denominator i.e. $\left|R_{\alpha, n}(z)\right| \rightarrow \infty$ as $z \rightarrow \infty$, so infinity is regarded as a fixed point. In addition, $\operatorname{deg} P>\operatorname{deg} Q+1$ where

$P=z^{n+1}+\alpha$ and $Q=2 z^{n}$, then the Julia set of $R_{\alpha, n}(z)$ is the boundary of the basin of attraction of infinity. In order to find the fixed points of the family $R_{\alpha, n}(z)=\frac{1}{2}\left(z+\frac{\alpha}{z}\right)$, we solve the equation

$z^{n+1}-\alpha=0$

The critical points are obtained by solving the equation

$$
z^{n+1}-\alpha n=0
$$

We can deduce that the family

$$
R_{\alpha, n}(z)=\frac{1}{2}\left(z+\frac{\alpha}{z^{n}}\right) \text { has } n+1 \text { critical points }
$$

excluding the one at infinity. $R$ has a pole of $\operatorname{order} n$ at the origin, the presence of a pole at the origin reveals that there is a neighborhood of the origin that is mapped to the basin at $\infty$. 
For clarity and simplicity we have generated the Julia sets of the family $R_{\alpha, 2}(z)=\frac{1}{2}\left(z+\frac{\alpha}{z^{2}}\right)$ as per Fig.1. One way to investigate Julia sets and their structure is to plot them using a computer programme. There are many ways of doing this and many programs available capable of producing quite intricate pictures [10]. The maps of Julia sets produced in this paper were generated on Matlab. We have assigned the complex parameter real value. Note that this family has critical points at the third roots of $2 \alpha$.

\section{Lemma 2.1}

If $n=2$, then $R_{\alpha, 2}(z)$ has a reflection about the imaginary axis.

Proof

$$
\begin{aligned}
& \text { Let } R_{\alpha, 2}(z)=\frac{1}{2}\left(z+\frac{\alpha}{z^{2}}\right) \\
& R_{\alpha, 2}(-z)=\frac{1}{2}\left(-z+\frac{\alpha}{z^{2}}\right)
\end{aligned}
$$

Adding member by member we get

$$
\begin{aligned}
& R_{\alpha, 2}(z)+R_{\alpha, 2}(-z)=\frac{\alpha}{z} \\
& \text { Therefore } R_{\alpha, 2}(-z)=-R_{\alpha, 2}(z)+\frac{\alpha}{z^{2}} .
\end{aligned}
$$

\section{Lemma 2.2}

If $n=2$, then $R_{\alpha, 2}(i z)$ has a reflection about the real axis.

\section{Proof}

Let

$R_{\alpha, 2}(i z)=\frac{1}{2}\left(i z-\frac{\alpha}{z^{2}}\right) ; R_{\alpha, 2}(-i z)=\frac{1}{2}\left(-i z-\frac{\alpha}{z^{2}}\right)$

Adding member by member in the above expressions we get

$$
R_{\alpha, 2}(i z)+R_{\alpha, 2}(-i z)=-\frac{\alpha}{z^{2}}
$$

Therefore $R_{\alpha, 2}(-i z)=-R_{\alpha, 2}(i z)-\frac{\alpha}{z^{2}}$. Apart from reflection, the family, $R_{\alpha, 2}(z)=\frac{1}{2}\left(z+\frac{\alpha}{z^{2}}\right)$ presents also some symmetry as shown in Fig.1, since it is the sum of identity and even functions.

The first derivative of the rational map $R_{\alpha, n}(z)$ is given by

$$
R_{\alpha, n}^{\prime}(z)=\frac{1}{2}\left(1-\frac{\alpha n}{z^{n+1}}\right)
$$

Let us now evaluate this derivative at infinity we get,

$$
R_{\alpha, n}^{\prime}(\infty)=\lim _{z \rightarrow \infty} R_{\alpha, n}^{\prime}(z)=\frac{1}{2},
$$

when this derivative is evaluated at zero we get,

$$
R_{\alpha, n}^{\prime}(0)=\lim _{z \rightarrow 0} R_{\alpha, n}^{\prime}(z)=\infty .
$$

Let us now define the rational map

$M_{\alpha, n}(z)=2 R^{\prime}(z)=1-\frac{\alpha n}{z^{n+1}}$

It is obvious that Eqn.(2.11) is the double of the first derivative of the rational map $R_{\alpha, n}(z)$ with $M_{\alpha, n}(\infty)=\lim _{z \rightarrow \infty} 2 R^{\prime}(z)=1$.

If $\mathrm{n}$ is an odd number, then $M_{\alpha, n}(-z)=M_{\alpha, n}(z)$. For quadratic rational maps, J. Milnor [11] suggested considering, among others, the family of those maps that take one critical point to the other. In appropriate coordinates these maps take the form $z \mapsto 1+\frac{1}{\omega z^{2}}$. Before the work of Milnor, the family

$f_{2}=\left\{z \rightarrow 1+\frac{1}{\omega z^{2}}: \omega \in C \backslash\{0\}\right\}$ was considered by M. Lyubich [12]. He asked whether the maps in this family have Herman rings. M. Shishikura [13], using quasi-conformal surgery techniques, proved that the quadratic rational maps have no Herman rings, thus solving Lyubich's question as a particular case.

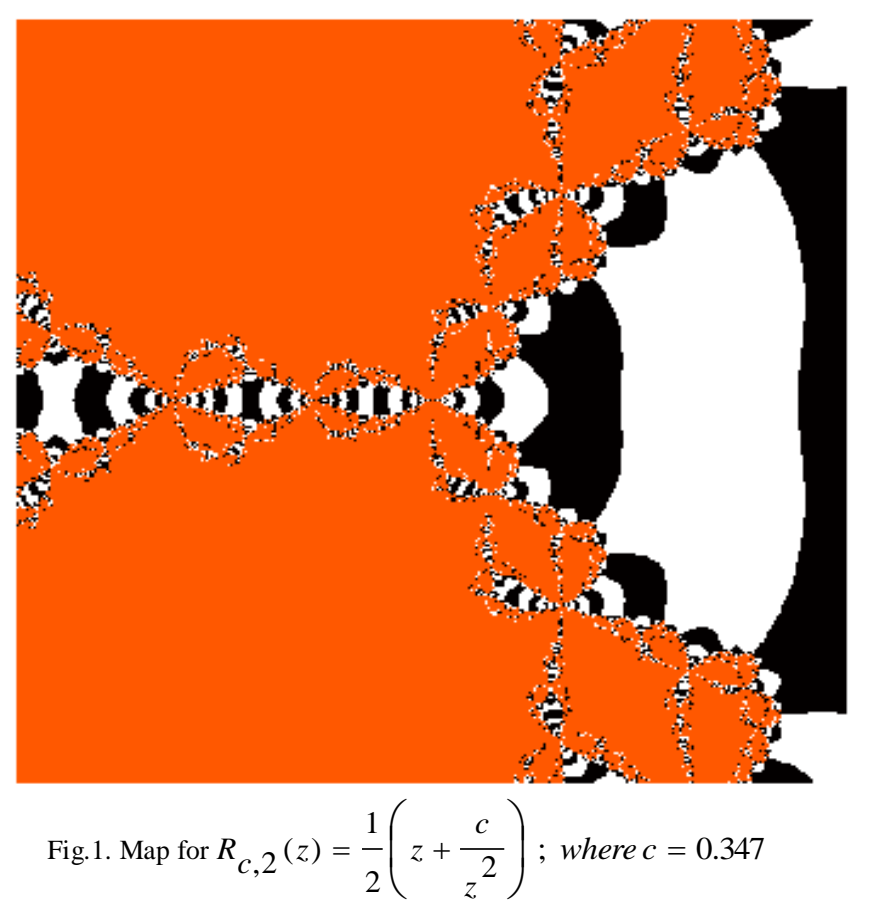


A component $U$ of the Fatou set $C \cup \infty \backslash J(f)$ is called a Herman ring if $U$ is comformally isomorphic to some annulus $A_{r}=\{z ; 1<|z|<r\}$, and if $f$ correspond to an irrational rotation of this annulus [14]. In [5], it was also proved that the family $f_{d}=\left\{z \rightarrow 1+\frac{1}{\omega z}: \omega \in C \backslash\{0\}\right\}$ has no Herman rings.

We can deduce that the rational map $M_{\alpha, n}(z)=1-\frac{\alpha n}{z^{n+1}}$ where $\omega=-\frac{1}{\alpha n}$ has no Herman rings. The Julia set of $M_{\alpha, n}(z)$ is shown in Fig.2.

\section{WAVELETS BASED ITERATED FUNCTIONS}

Wavelets find applications and have significant impact in various scientific areas including geophysics, hydrodynamics, econometrics, data processing, image compression, detection of discontinuities, neural networks, etc [15].

One of the reasons wavelets have found so many uses and applications is that they are especially attractive from the computational point of view.

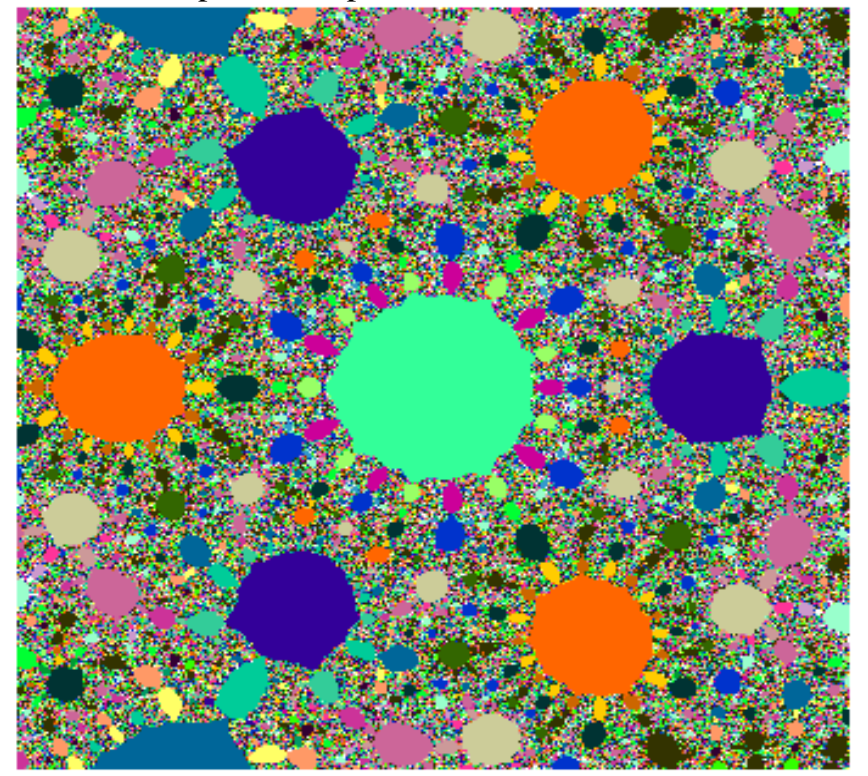

$$
\text { Fig.2.Map for } M_{\alpha, 2}(z)=1-\frac{2 \times c}{z^{3}} ; \text { where } c=\tan \frac{\pi}{3}
$$

Computational efficiency of wavelets lies in the fact that wavelet coefficients in wavelet expansions for functions in $V_{0}$ (resolution subspace in $L^{2}\left(R^{d}\right)$ may be computed using matrix iteration, rather than by a direct computation of inner products: the latter would involve integration over $R^{d}$, and hence be computationally inefficient, if feasible at all. The deeper reason for why we can compute wavelet coefficients using matrix iteration is an important connection to the subband filtering method from signal/image processing involving digital filters, down-sampling and up-sampling. In this setting filters may be realized as functions $m_{0}$ on a dtorus, e.g., quadrature mirror filters [16]. It would be interesting to adapt and modify the Haar wavelet, and the other wavelet algorithms to the Julia sets [17].

One of the applications of these IFSs, and their spectral theory, is to image processing [18] and [19]. IFSs include dynamical systems defined from a finite set of affine and contractive mappings in $R^{d}$, or from the branches of inverses of complex polynomials, or of rational mappings in the complex plane. A unifying approach to wavelets, dynamical systems, iterated function systems, self-similarity and fractals may be based on the systematic use of operator analysis and representation theory [20].

In terms of signal processing, what the two have in common, wavelets and IFSs, is that large scale data may be compressed into a few functions or parameters. In the case of IFSs, only a few matrix entries are needed, and a finite set of vectors in $R^{d}$ must be prescribed. As is shown in [18], this can be turned into effective codes for large images. Similarly (see [21]) discrete wavelet algorithms can be applied to digital images and to data mining. The efficiency in these applications lies in the same fact: The wavelets may be represented and determined by a small set of parameters; a choice of scaling matrix and of masking coefficients, i.e., the coefficients $a_{k}$ in the scaling identity Eqn. (3.1) below [22]. The scaling function $\varphi$ satisfies an important equation, called the scaling equation. This is obtained by considering the function $\varphi\left(A^{-1}\right)$ which lies in $V_{-1} \subset V_{0}$ Since the translates of $\varphi$ form a basis for $V_{0}$, the scaling function is obtained:

$$
\begin{aligned}
& \frac{1}{\sqrt{|\operatorname{det} A|}} \varphi\left(A^{-1} x\right) \\
& =\sum_{k \in Z^{d}} a_{k} \varphi(x-k), \quad\left(x \in R^{d}\right)
\end{aligned}
$$

where $\left(a_{k}\right)_{k \in Z}$ is a sequence of complex numbers.

\section{Definition 3.1}

Let $z \mapsto R(z)$ be a given polynomial. Let $\mathrm{P}$ be a finite set of distinct polynomials each of degree less than the degree of R. Let $K^{2}(R, P)$ be a space of functions $\mathrm{F}$ in $H^{2}(J(R))$. We say that $\mathrm{F}$ is in $K^{2}(R, P)$ if there are functions $F_{p}(z)$ in $H^{2}(J(R))$ indexed by $\mathrm{P}$ such that $F(z)=\sum_{p \in P} p(z) F_{p}(R(z))$

It is easy to see that each $K^{2}(R, P)$ is a closed subspace in $H^{2}(J(R))$, so in particular it is a Hilbert 
space. The finite family $\mathrm{P}$ that enters Eqn.(3.2) is a family of generalized filters. It depends on the particular polynomial $z \mapsto R(z)$, and we can expect to find solutions to Eqn. (3.2) in Definition 3.1 from the kind of representations of the Cuntz algebras the authors studied in their work on wavelets on fractals [19].

In this paper we will deal with the complex iteration systems which generate Julia sets in the Riemann sphere.

\section{SIMULATION WITH SPTOOL}

In this section we are interested in complex signals generated by importing the Julia set shown in Fig.1, from Matlab workspace to matlab signal processing tool with the sampling frequency of $8192 \mathrm{~Hz}$. This tool has three main components: signal section view, filter section view and edit as well as spectra section. These are used to visualize waveforms and spectra of several signals and make a qualified filter design. In order to use a complex signal under these processes the Julia fractal was imported as an array of 400 column index vectors, each of them having the real part, imaginary part, magnitude and phase angle. Fig.3, Fig.4, Fig.5 and Fig.6 show the sampled real part, imaginary part, magnitude and phase angle of the column index vector 1 respectively.

Let $x(t)=\left[\begin{array}{lllll}x_{1}(t) & x_{2}(t) & . & . & x_{m}(t)\end{array}\right]$ be the sampling vector signal

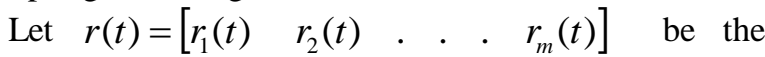
sampled vector signal

Let $y(t)=\left[\begin{array}{llllll}y_{1}(t) & y_{2}(t) & . & . & . & y_{m}(t)\end{array}\right]$ be the response of the band pass filter

Let $f(t)$ be the impulse response of the band pass filter

Let $J=\left[\begin{array}{cccccc}J_{11} & J_{12} & \cdot & \cdot & \cdot & J_{1 m} \\ J_{21} & J_{22} & \cdot & \cdot & \cdot & J_{2 m} \\ \cdot & \cdot & \cdot & \cdot & \cdot & \cdot \\ \cdot & \cdot & \cdot & \cdot & \cdot & \cdot \\ \cdot & \cdot & \cdot & \cdot & \cdot & \cdot \\ J_{m 1} & J_{m 2} & \cdot & \cdot & \cdot & J_{m m}\end{array}\right]$ be $m \times m$ data

matrix representing the Julia set $J(f)$ in Fig.1. This data matrix is a complex matrix, $J \in Z^{m \times m}$, associated with $m \times m$ Pixels of the Julia set. In our case we have considered $m=400$ in the Matlab programme that has generated the Julia set. The Matlab signal processing tool shows that the sampled vector signal generated out of the Julia set $J(f)$ is a complex signal with real part, imaginary part, magnitude and phase. In this section we attempt to express the sampled vector signal $r(t)$ in terms of sampling vector signal $x(t)$ as well as data matrix $J$ representing the Julia set.

$$
\begin{aligned}
& r_{1}(t)=J_{11} x_{1}(t)+J_{21} x_{2}(t)+\ldots+J_{m 1} x_{m}(t)=\sum_{i=k=1}^{m} J_{i 1} x_{k}(t) ; \\
& \left|r_{1}(t)\right|=\left|\sum_{i=k=1}^{m} J_{i 1} x_{k}(t)\right| \\
& r_{2}(t)=J_{12} x_{1}(t)+J_{22} x_{2}(t)+\ldots+J_{m 2} x_{m}(t)=\sum_{i=k=1}^{m} J_{i 2} x_{k}(t) ; \\
& \left|r_{2}(t)\right|=\left|\sum_{i=k=1}^{m} J_{i 2} x_{k}(t)\right| \\
& r_{m}(t)=J_{1 m} x_{1}(t)+J_{2 m} x_{2}(t)+\ldots+J_{m m} x_{m}(t)=\sum_{i=k=1}^{m} J_{i m} x_{k}(t) ; \\
& \left|r_{m}(t)\right|=\left|\sum_{i=k=1}^{m} J_{i m} x_{k}(t)\right|
\end{aligned}
$$

Since $r(t)=x(t) \times J$ is a complex signal, then, the magnitude of the sampled signal is given by:

$$
\begin{aligned}
|r(t)| & =\sqrt{r_{1}{ }^{2}(t)+r_{2}{ }^{2}(t)+\ldots+r_{m}{ }^{2}(t)} \\
& =\sqrt{\sum_{j=1}^{m}\left(\sum_{i=k=1}^{m} J_{i j} x_{k}(t)\right)^{2}}
\end{aligned}
$$

In vector form $r(t)=x(t) \times J$ can be written as:

$$
\begin{aligned}
& {\left[\begin{array}{c}
r_{1}(t) \\
r_{2}(t) \\
\cdot \\
\cdot \\
r_{m}(t)
\end{array}\right]=\left[\begin{array}{cccccc}
J_{11} & J_{21} & \cdot & \cdot & \cdot & J_{m 1} \\
J_{12} & J_{22} & \cdot & \cdot & \cdot & J_{m 2} \\
\cdot & \cdot & \cdot & \cdot & \cdot & \cdot \\
\cdot & \cdot & \cdot & \cdot & \cdot & \cdot \\
\cdot & \cdot & \cdot & \cdot & \cdot & \cdot \\
J_{1 m} & J_{2 m} & \cdot & \cdot & \cdot & J_{m m}
\end{array}\right] \times\left[\begin{array}{c}
x_{1}(t) \\
x_{2}(t) \\
\cdot \\
\cdot \\
\cdot \\
x_{m}(t)
\end{array}\right]} \\
& r^{T}(t)=J^{T} \times x^{T}(t)=(x(t) \times J)^{T}
\end{aligned}
$$

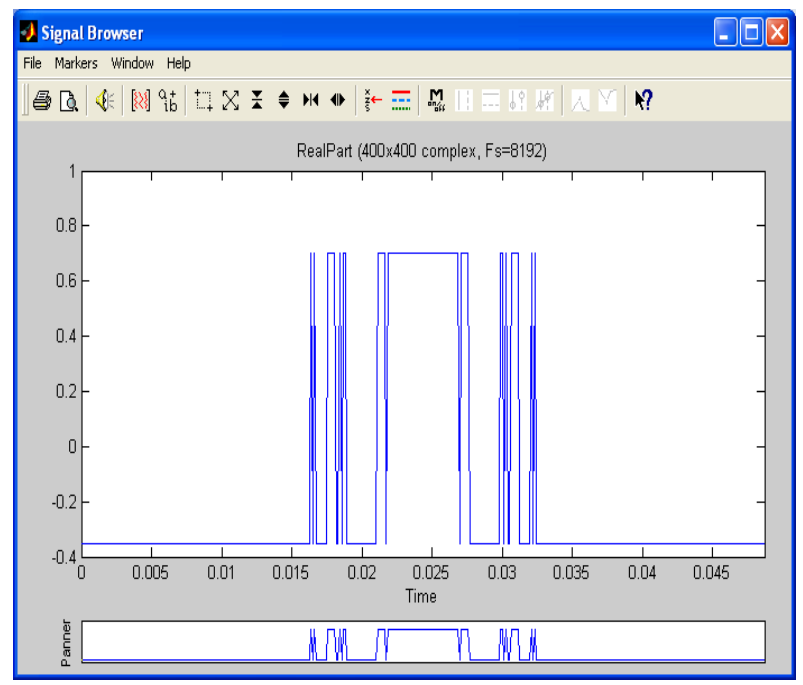

Fig.3. Real Part for Column Index Vector 1 


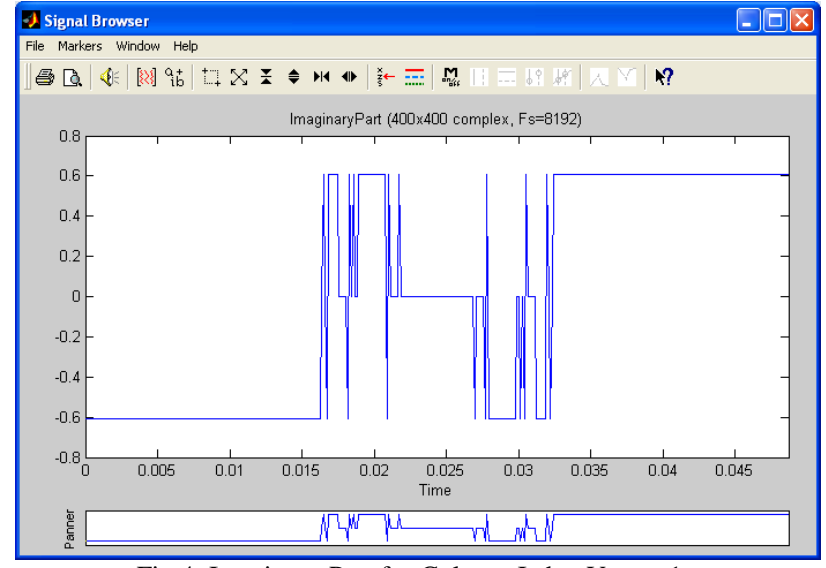

Fig.4. Imaginary Part for Column Index Vector 1

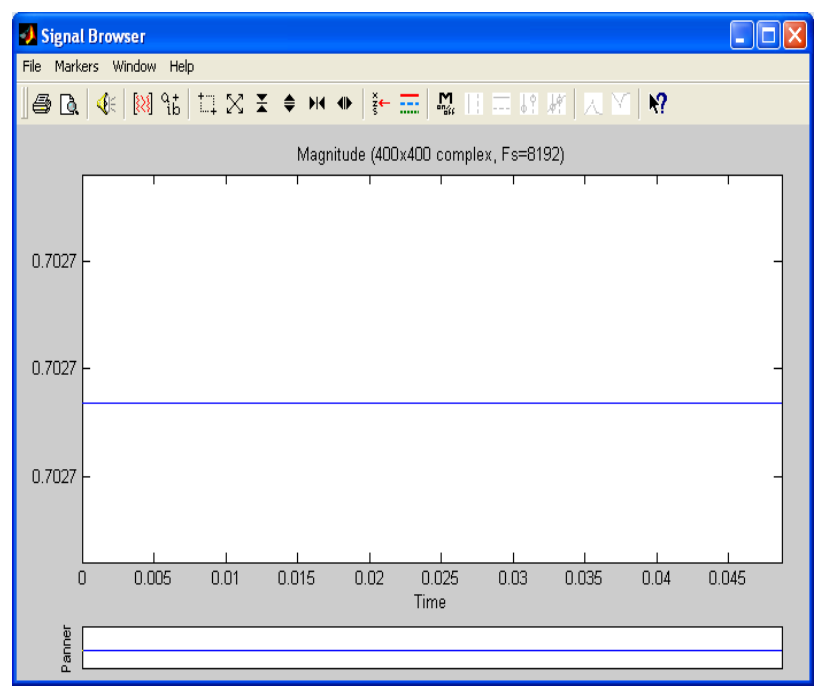

Fig.5. Magnitude for Column Index Vector 1

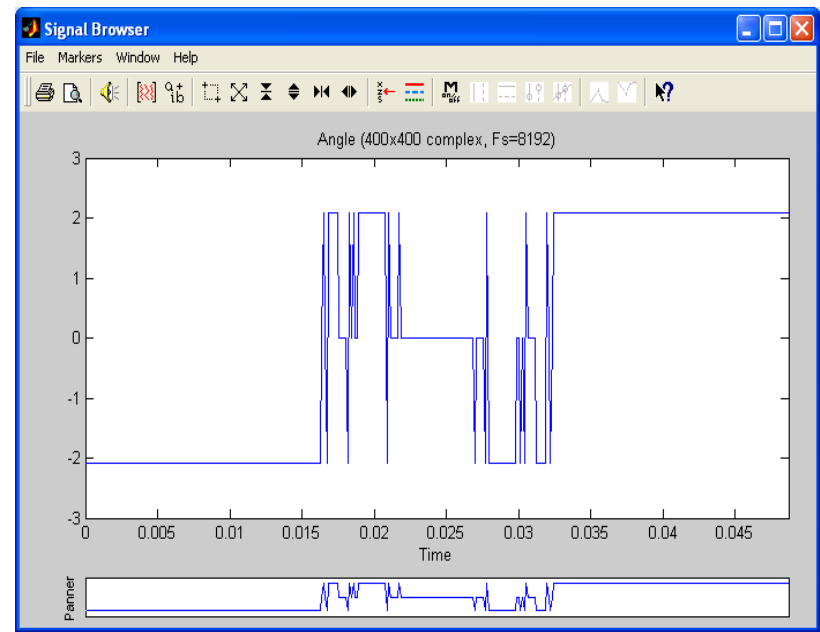

Fig.6. Angle for Column Index Vector 1

The power spectral density (PSD) of the sampled complex signal is shown in Fig.7. A stable band pass filter has been designed to analyze the complex signal in the sampling frequency range. Fig.8 shows magnitude, phase response, step response, group phase delay and other properties whereas Fig.9 indicates the pole-zero plot of the filter. The filtered real part, imaginary part, magnitude and phase angle of the column index vector 1 are shown in Fig.10, Fig.11, Fig.12 and Fig.13 show respectively. The filtering process has eliminated noise successfully. The power spectral density (PSD) of the filtered complex signal is shown in Fig.14. By definition the response of the band pass filter is given by the convolution of the impulse response $f(t)$ of the band pass filter with the sampled vector signal $r(t)=\left[\begin{array}{llllll}r_{1}(t) & r_{2}(t) & . & . & r_{m}(t)\end{array}\right]$. Hence we get $y(t)=f(t) * r(t)=\int r(t) f(t-\sigma) d \sigma$.

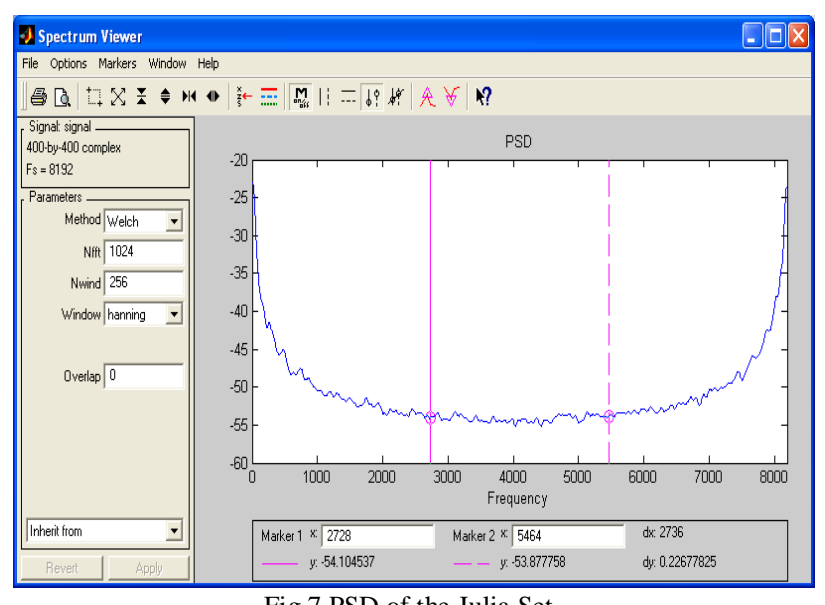

Fig.7.PSD of the Julia Set

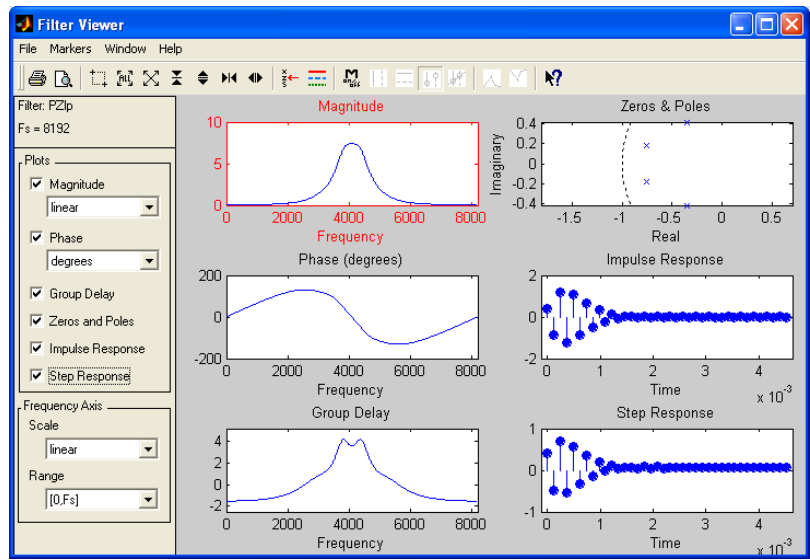

Fig.8. Band Pass Filter Characteristics

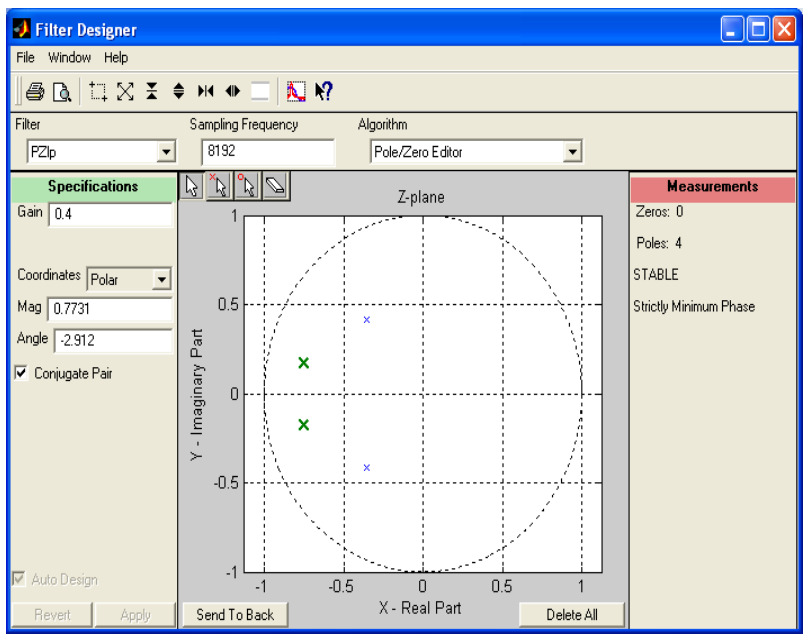

Fig.9. Band Pass Filter Pole-Zero Plot 
Apply the definition to each column, we get:

$$
\begin{aligned}
& y_{2}(t)=r_{2}(t) * f(t)=\int r_{2}(t) f(t-\sigma) d \sigma \\
& =\int\left(\sum_{i=k=1}^{m} J_{i 2} x_{k}(t)\right) f(t-\sigma) d \sigma \\
& =\sum_{i=k=1}^{m} J_{i 2} \int x_{k}(t) f(t-\sigma) d \sigma \\
& y_{1}(t)=r_{1}(t) * f(t)=\int r_{1}(t) f(t-\sigma) d \sigma \\
& =\int\left(\sum_{i=k=1}^{m} J_{i 1} x_{k}(t)\right) f(t-\sigma) d \sigma \\
& =\sum_{i=k=1}^{m} J_{i 1} \int x_{k}(t) f(t-\sigma) d \sigma
\end{aligned}
$$$$
y_{m}(t)=r_{m}(t) * f(t)=\int r_{m}(t) f(t-\sigma) d \sigma
$$

$$
\begin{aligned}
& =\int\left(\sum_{i=k=1}^{m} J_{i m} x_{k}(t)\right) f(t-\sigma) d \sigma \\
& =\sum_{i=k=1}^{m} J_{i m} \int x_{k}(t) f(t-\sigma) d \sigma
\end{aligned}
$$

$y_{j}(t)=w_{s_{j}, \tau_{j}}(t)=\sum_{i=k=1}^{m} J_{i j} \int x_{k}(t) f(t-\sigma) d \sigma$

$$
\begin{aligned}
& =\sum_{i=k=1}^{m} J_{i j} \int x_{k}(t) f(t-\sigma) d \sigma \\
& =\left[\cos \omega_{0}\left(\frac{t-\tau_{j}}{s_{j}}\right)+j \sin \omega_{0}\left(\frac{t-\tau_{j}}{s_{j}}\right)\right] h_{s_{j}, \tau_{j}}(t)
\end{aligned}
$$

where

$$
h_{s_{j}, \tau_{j}}(t)=\frac{1}{\sqrt{s_{j}}} e^{-\frac{1}{2}\left(\frac{t-\tau}{s_{j}}\right)^{2}}
$$

The complex wavelet generated out of the Julia set of $m \times m$ Pixels, is given by:

$$
w_{s, \tau}=\left\lfloor\begin{array}{llllll}
w_{s_{1}, \tau_{1}}(t) & w_{s_{2}, \tau_{2}}(t) \quad . \quad . \quad . & w_{s_{m}, \tau_{m}}(t)
\end{array}\right]
$$

$$
\begin{aligned}
w_{s_{1}, \tau_{1}}(t)= & \sum_{i=k=1}^{m} J_{i 1} \int x_{k}(t) f(t-\sigma) d \sigma \\
= & {\left[\cos \omega_{0}\left(\frac{t-\tau_{1}}{s_{1}}\right)+j \sin \omega_{0}\left(\frac{t-\tau_{1}}{s_{1}}\right)\right] h_{s_{1}, \tau_{1}}(t) } \\
w_{s_{2}, \tau_{2}}(t)= & \sum_{i=k=1}^{m} J_{i 2} \int x_{k}(t) f(t-\sigma) d \sigma \\
= & {\left[\cos \omega_{0}\left(\frac{t-\tau_{2}}{s_{2}}\right)+j \sin \omega_{0}\left(\frac{t-\tau_{2}}{s_{2}}\right)\right] h_{s_{2}, \tau_{2}}(t) } \\
w_{s_{m}, \tau_{m}}(t)= & \sum_{i=k=1}^{m} J_{i m} \int x_{k}(t) f(t-\sigma) d \sigma \\
= & {\left[\cos \omega_{0}\left(\frac{t-\tau_{m}}{s_{m}}\right)+j \sin \omega_{0}\left(\frac{t-\tau_{m}}{s_{m}}\right)\right] h_{s_{m}, \tau_{m}}(t) }
\end{aligned}
$$

The magnitude of the complex wavelet generated out of the Julia set of $m \times m$ Pixels, is given by:

$$
\begin{aligned}
& \left|w_{s_{1}, \tau_{1}}(t)\right|=h_{s_{1}, \tau_{1}}(t)=\frac{1}{\sqrt{s_{1}}} e^{-\frac{1}{2}\left(\frac{t-\tau_{1}}{s_{1}}\right)^{2}} \\
& \left|w_{s_{2}, \tau_{2}}(t)\right|=h_{s_{2}, \tau_{2}}(t)=\frac{1}{\sqrt{s_{2}}} e^{-\frac{1}{2}\left(\frac{t-\tau_{2}}{s_{2}}\right)^{2}} \\
& \left|w_{s_{m}, \tau_{m}}(t)\right|=h_{s_{m}, \tau_{m}}(t)=\frac{1}{\sqrt{s_{m}}} e^{-\frac{1}{2}\left(\frac{t-\tau_{m}}{s_{m}}\right)^{2}}
\end{aligned}
$$

The phase of the complex wavelet generated out of the Julia set of $m \times m$ Pixels, is given by:

$\phi_{s, \tau}=\left\lfloor\begin{array}{lllll}\phi_{s_{1}, \tau_{1}}(t) & \phi_{s_{2}, \tau_{2}}(t) & \cdot & \cdot & \phi_{s_{m}, \tau_{m}}(t)\end{array}\right]$

$$
\begin{aligned}
& \phi_{s_{1}, \tau_{1}}(t)=\frac{t-\tau_{1}}{s_{1}} \\
& \phi_{s_{2}, \tau_{2}}(t)=\frac{t-\tau_{2}}{s_{2}} \\
& \phi_{s_{m}, \tau_{m}}(t)=\frac{t-\tau_{m}}{s_{m}}
\end{aligned}
$$


We can repeat the same steps on the rational map $M_{\alpha, 2}(z)=1-\frac{2 \times \tan \frac{\pi}{3}}{z^{3}}$ shown in Fig. 2 .

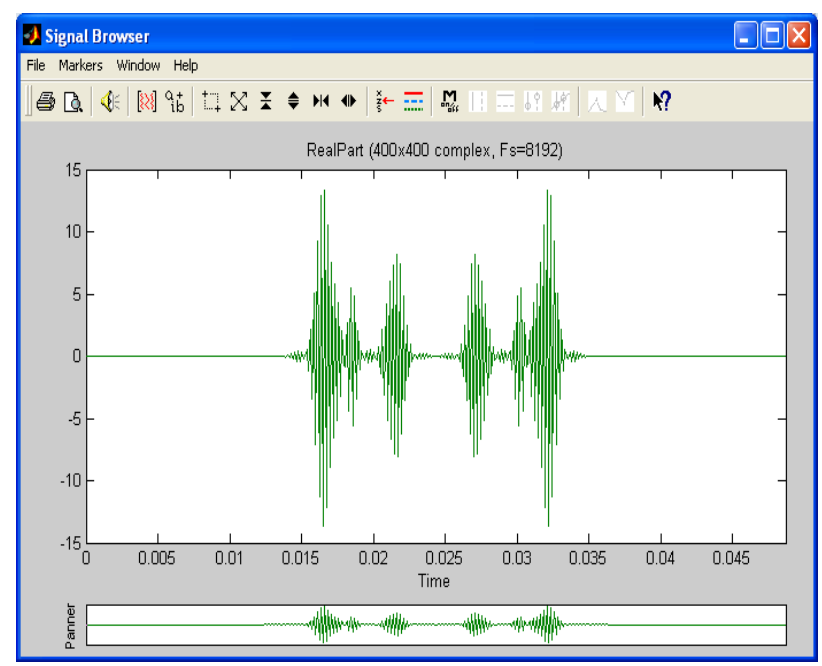

Fig.10. Filtered Real Part for Column Index Vector 1

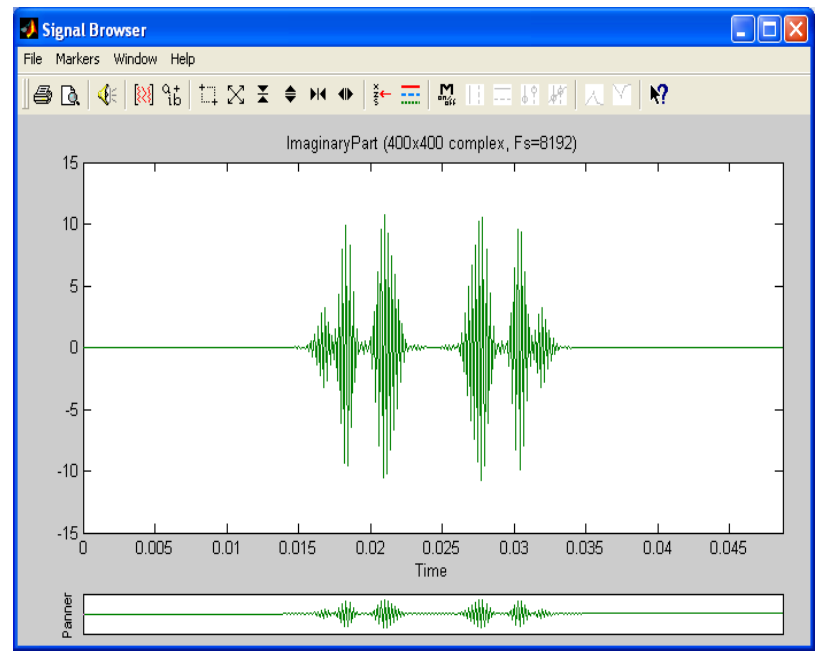

Fig.11. Filtered Imaginary Part for Column Index Vector 1

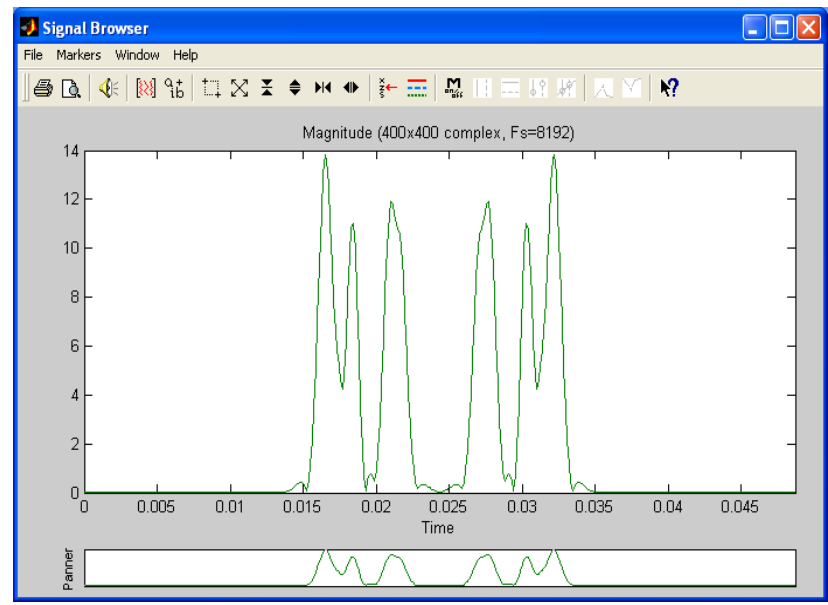

Fig.12. Filtered Magnitude for Column Index Vector 1

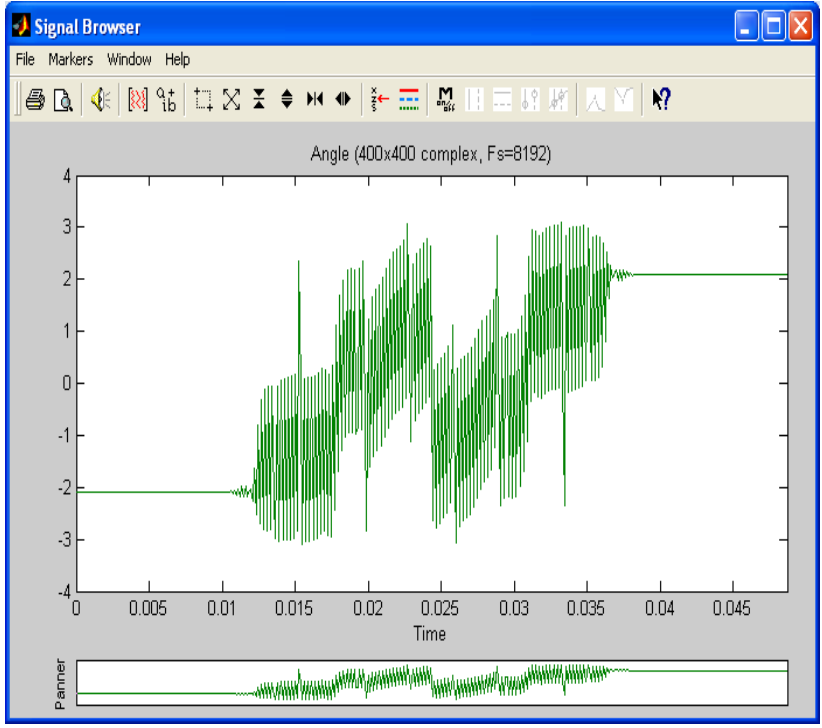

Fig.13. Filtered Angle for Column Index Vector 1

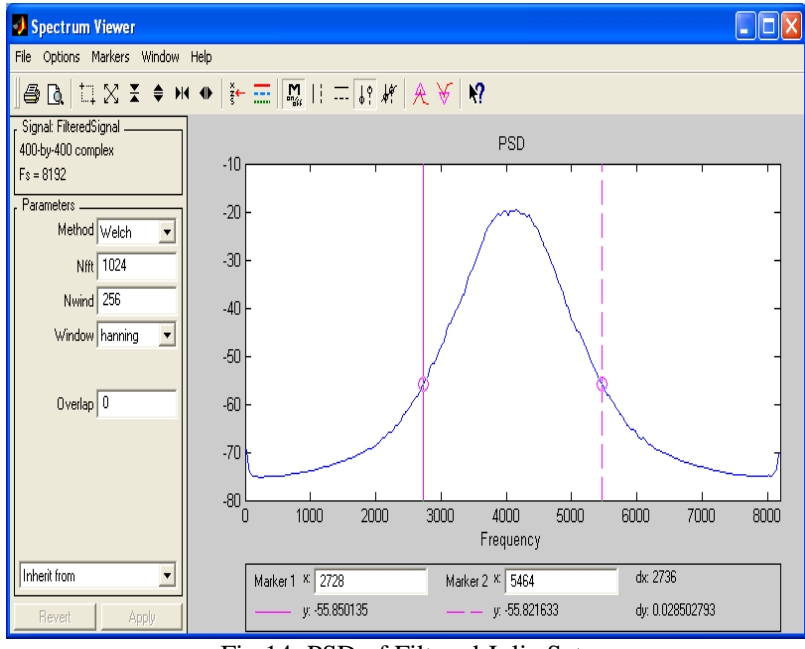

Fig.14. PSD of Filtered Julia Set

\section{CONCLUSION}

This research aimed at the generation of wavelets in the dynamics of rational maps having Zhukovskii function. But the reasoning applied on these rational maps can be extended to any other rational map. Indeed we have compute Julia sets of the rational maps with $400 \times 400$ pixels for 500 iterations using Matlab programme. We have then imported the data associated with the Julia set from Matlab workspace to Signal Processing Tool and generated complex signals through the sampling process of $8192 \mathrm{~Hz}$. The filtering process of these complex signals has eliminated noise successfully. The effect of the filter has generated wavelets on both real part and imaginary part of the complex signals generated by the Julia set. We have also found that the filtered complex signals have dominating frequency at half of the sampling frequency. 


\section{REFERENCES}

[1] Jean-Bosco Mugiraneza, On Computing Some Julia Sets of Rational Maps Having Zhukovskii Function, Canadian Journal on Computing in Mathematics, Natural Sciences, Engineering and Medicine, Vol. 2 $\mathrm{N}^{\mathrm{o}}$. 7, pp: 187-202, August 2011, ISSN: 1923-1660, AM Publishers Corporation, Canada

[2] Dynamics of Rational Functions, Graduate Lecture Course, University of Bristol, Spring 2007

[3] Curtis T. McMullen, Frontiers in Complex Dynamics, University of California, Berkeley CA 94720, 16 February, 1994

[4] Alan F. Beardon, Iteration of Rational Functions: Complex Analytic Dynamical Systems. Number 132 in Graduate Texts in Mathematics. SpringerVerlag, New York, 1991.

[5] R. Bamón and J. Bobenrieth, The Rational Maps $z \mapsto 1+\frac{1}{\omega z} d$ Have no Herman Rings, Proceedings of the American Mathematical Society, Volume 127, Number 2, February 1999, Pages 633636 S 0002-9939(99)04566-9

[6] Robert L. Devaney, Dynamics of $z^{n}+\frac{\lambda}{z^{n}}$, Why the Case $n=2$ is Crazy, Boston University, February 2011

[7] D.J. Acheson, Elementary Fluid Dynamics, Clarendon Press, Oxford, UK, 1990

[8] Rachel W. Hall and Krešimir Josić, Planetary Motion and the Duality of Force Laws, SIAM, Vol. 42, No.1, pp.115-124, AMS:70F05,30A99,30C99, 2000

[9] Robert L. Devaney, Matt Holzer, and David Uminsky, Blow Up Points and Baby Mandelbrot Sets for Singularly Perturbed Rational Maps, 1991, Mathematical Subject Classification. Primary 37F45; Secondary 37F10.

[10] Jonathan Fraser, An Introduction to Julia Sets, April 16, 2009

[11] J. Milnor, Geometry and Dynamics of Quadratic Rational Maps, Experimental Mathematics (1) 2 (1993), 37 - 83. MR 96b:58094

[12] M. Lyubich, The dynamics of Rational Transforms: The Topological Picture, Russian Math. Surveys (4) 41 (1986), 3595. MR 88g: 58094

[13] M. Shishikura, On the Quasiconformal Surgery of Rational Functions, Ann. Sci. Ec. Norm. Sup. 20 (1987), 1 - 29. MR 88i:58099

[14] J. Milnor, Dynamics in One Complex Variable, $3^{\text {rd }}$ Edition, Princeton University Press, 2006

[15] Shahriar Yousefi, Ilona Weinreich and Dominik Reinarz, Wavelet-Based Prediction of Oil Prices, Chaos Solitons and Fractals, Elsevier, November 2004

[16] Dorin Ervin Dutkay and Palle E.T. Jorgensen, Iterated Function Systems, Ruelle Operators, and Invariant Projective Measures, March 2008
[17] Palle E.T. Jorgensen and Myung-Sin Song, Comparison of Discrete and Continuous Wavelet Transforms, July 2007

[18] Michael Fielding Barnsley, Superfractals. Cambridge University Press, Cambridge, 2006.

[19] Dorin E. Dutkay and Palle E. T. Jorgensen, Wavelets on Fractals. Rev. Mat. Iberoamericana, 22(1):131-180, 2006.

[20] Myung-Sin Song, Analysis of Fractals, Image Compression and Entropy Encoding, Southern Illinois University Edwards ville, July 10, 2009

[21] Palle E. T. Jorgensen, Analysis and probability: Wavelets, Signals, Fractals, volume 234 of Graduate Texts in Mathematics, Springer, New York, 2006.

[22] Dorin Ervin Dutkay and Palle E.T. Jorgensen, Fourier Series On Fractals: A Parallel with Wavelet Theory, January 2008

\section{AUTHOR}

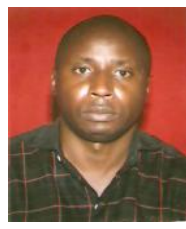

Mr. Jean-Bosco Mugiraneza received the BSc in Electromechanical Engineering in 2003 from Kigali Institute of Science and Technology (KIST) and the M.E. in Electrical Engineering in 2006 from City University of New York. Mr. Jean Bosco is Member of International Association of Engineers (MIAENG), Member of International Association of Computer Science and Information Technology (MIACSIT), Member of International Association of Science and Technology for Development (MIASTED) as well as Member of International Society for Engineering Education (MIGIP). He is the co-author of the books on Principles of Engineering Analysis, ISBN: 9788184871456 and Signals and System Analysis with Matlab and PSpice, ISBN: 978384336681. 